\title{
DESARROLLO Y SIMULACIÓN DE UNA ESTACIÓN BASE GSM/CDMA UTILIZANDO SOFTWARE RADIO
}

\author{
Julián Busqués V. ${ }^{1} \quad$ Héctor Reyes S..$^{1} \quad$ Juan Hernández S. ${ }^{1}$
}

Recibido 19 de julio de 2002, aceptado el 10 de noviembre de 2002

\begin{abstract}
RESUMEN
Se presentan los aspectos más relevantes del diseño de una estación genérica multiestándar GSM/CDMA en base a la a de Software Radio. Primeramente se presentan conceptos de esa tecnología. Se describe luego el diseño de la estación base, el estudio simulado de su implementación protot pica.
\end{abstract}

Palabras claves: Software Radio. telefonía móvil, esquemas GSM y CDMA, ope

\begin{abstract}
The more relevant aspects of the design of a generic multistandard GSM/CDMA station, based upon the new Software Radio technology, are presented. Some concepts of that technology are presented first. The design of the base station is described next, followed by a simulation of a prototypical implementation.
\end{abstract}

Keywords: Radio Software, mobile telephony, GSM and CDMA outlines, GSM/CDM operation

\section{INTRODUCCIÓN}

En la actualidad existen diversas tecnologías en telefonía móvil y distintas empresas que trabajan con ellas. En este trabajo interesa el caso de empresas en Chile y las tecnologías GSM (Global System for Mobile Communications) y CDMA (Code Division Multiple Access), [1] y [2]. Cada empresa instala sus propias estaciones base, de acuerdo a la tecnología utilizada y al espectro de frecuencias que usa, lo que lleva a una proliferación de antenas y a una serie de problemas urbano-ambientales.

En este trabajo se plantea el desarrollo y simulación de una estación base, de telefonía móvil, que puede ser utilizada por dos, o más, empresas que empleen tecnologías diferentes pero que trabajen en el mismo espectro de frecuencias autorizadas. Los diseños y desarrollos elaborados se basan en la novel disciplina de Software Radio (SR), o Software-Defined Radio, términos introducidos por J. Mitola, [3]-[6].

A continuación se presentan los conceptos básicos de Software de Radio. En seguida se describe el diseño, el desarrollo y la simulación de una estación base genérica multiestándar GSM/CDMA, empleando la Software Radio. El trabajo está basado en la referencia
[7] de los autores, donde se describen detalladamente aspectos de la telefonía móvil, su situación en Chile, y los circuitos y programas desarrollados.

\section{SOFTWARE RADIO}

La tecnología de Software Radio fue iniciada por las fuerzas armadas de EE.UU. para conseguir comunicaciones permanentes en distintas bandas con un solo equipo, asegurar compatibilidad entre sistemas de comunicación de las diversas ramas (ejército, armada, fuerza aérea), lograr adaptabilidad frente a nuevas innovaciones de componentes y equipos, y para otros objetivos. Básicamente, se trata de trasladar a software muchos de las funciones que se efectúan hasta ahora en hardware. En el ámbito civil se denomin Software-Defined Radio.

Cada día se hace más complejo conjugar distintos tipos de sistemas de comunicación. La aparición, casi en forma continua, de nuevos avances y mejorías en los sistemas ya existentes hace imperiosa la necesidad de disminuir costos en la adaptación e instalación de nuevos métodos de transmisión/recepción de los mismos.

\footnotetext{
${ }^{1}$ Universidad Técnica Federico Santa María, Departamento de Electrónica, Casilla 110-V, Valparaíso - Chile, jhs@elo,utfsm.cl
} 
Dado que la mayor parte de los costos se encuentran en el hardware de las máquinas, que es de baja adaptación, es importante reemplazar estos elementos de hardware por unidades de software que permitan reducir los costos inmediatos y también permitir la incorporación de nuevos avances, u operadores de sistemas, agregando un mínimo de hardware y reemplazando este último por software que solucione el problema.

Un sistema de Software Radio consta básicamente de los subsistemas que se bosquejan en la Fig. 1, y que se basan en procesadores o en arreglos de puertas de campos programables FPGA (Field Programmable Gate Arrays), [4].

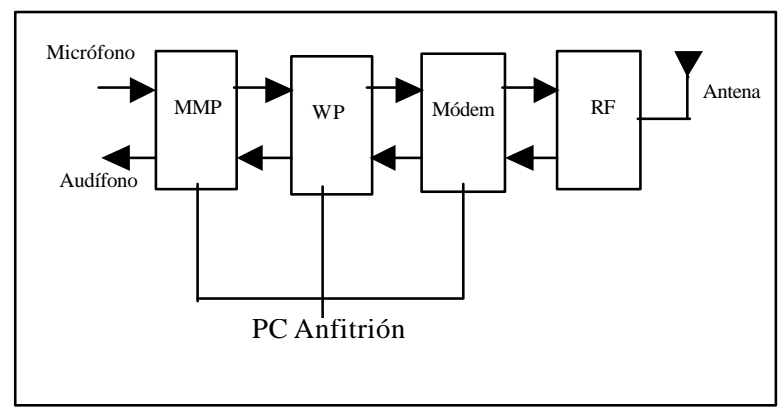

Fig. 1.- Esquema de Software-Radio estándar, en donde MMP, procesador multimedial; WP, procesador de formas de onda; RF, componentes de radio en hardware

En Fig. 1. el bloque RF es tradicional en sistemas de comunicaciones y contiene conversores de subida y bajada y el conmutador Tx/Rx. Se describirán, a continuación, las funciones de los otros cuatro subsistemas, que son propiamente de software radio.

\section{PC anfitrión (host)}

Este elemento es un microcomputador personal que contiene en software los MMP, WP y módem indicados en la Fig. 1. Este es el controlador maestro de todos los otros subsistemas e inicializa y monitorea las tareas del MMP, WP y el módem. Cumple además la labor de interfaz entre la máquina y el hombre (MMI, Man Machine Interface).

En el control del MMP y WP la labor del PC es fundamental, pues debe crear el medio, cargar y ejecutar los softwares de estos subsistemas, permitir la comunicación entre el MMP y el WP en el sentido correcto, según se esté transmitiendo o recibiendo información, monitorear los datos intercambiados por estos subsistemas, servir de tampón para estos datos y activar las herramientas necesarias para su análisis. Con respecto al módem, el PC carga los firmwares del FPGA y los softwares necesarios para el correcto funcionamiento de ese sistema.

\section{Procesador multimedial MMP}

Este subsistema realiza el procesamiento de las entradas y salidas de voz y datos en los modos de transmisión y de recepción. En cualquiera de estos modos el software del MMP ejecuta los codificadores de voz ("vocoder") deseado o el algoritmo de decodificación de formas de ondas, siendo estas últimas de voz o datos. También puede filtrar datos, cambiar la razón de adquisición de ellos, variar la ganancia de entrada y la atenuación de salida, y todas estas variables son programables en este subsistema. El MMP debe ser capaz de procesar en tiempo real las entradas y salidas, sean estas análogas o digitales. El software del MMP tiene por misión también la documentación con el PC y monitorear los cambios de transmisión a recepción, y viceversa.

\section{Procesador de formas de ondas WP}

Este subsistema tiene como misiones la generación de formas de onda y la recuperación de datos adquiridos. Debe ser capaz de realizar sus operaciones en tiempo real, y sus funciones son en transmisión y recepción. En modo Transmisión se reciben los datos que vienen desde el MMP, los que pasan por el PC. Para las frecuencias esperadas se crean las formas de ondas correspondientes. El subsistema sabe que forma de onda crear ya que en su memoria se encuentran tablas con código/frecuencia que el receptor (del WP) reconoce a priori. Para las frecuencias deseadas, las formas de onda son moduladas. Estos datos son enviados en múltiples tramas, con un salto sincronizado entre cada trama. El receptor utiliza un código inserto en estas tramas para ser habilitado, lo que permite sincronizar y correlacionar los datos que arriban.

En modo Recepción los datos que llegan al WP provienen del módem. Típicamente, este proceso se puede dividir en dos submodos, que son usados indistintamente de la implementación específica de la forma de onda. El método de implementación de estos submodos es dependiente de las especificaciones de las distintas formas de ondas y es implementado en software para apoyar futuros cambios de las mismas.

\section{Módem}

La estructura del subsistema Módem bosquejada en Fig.1 es relativamente compleja, y se basa en FPGA, como se indicó.

Contiene un módem (modulador/demodulador) e interfaces de control de frecuencia y de comunicación con los puertos de un Procesador Digital de Señales 
(DSP). Se asocia, vía conversores A/D y D/A, y control de RF, con el bloque RF y con un reloj maestro y una memoria RAM. La parte transmisora recibe los datos desde el WP, los que son puestos en tramas, procesados por el DSP y enviados al módem, el que modula estas tramas para traspasarlas al sistema de RF. En la parte receptora estos datos son recibidos por el sistema de RF y convertidos de análogo a digitales. La señal llega en alta frecuencia y es trasladada a una frecuencia en banda base (de varios órdenes de magnitud menor). Estos datos son demodulados y entran en banda base al DSP para ser procesados. Todo este proceso consta de la supervisión, entrega de información y análisis de datos de parte del PC.

\section{DISEÑO DE LA ESTACIÓN BASE}

Se presenta el diseño sugerido para la estación base multiestándar, que ha de funcionar para ambas tecnologías citadas, CMDA y GSM. Las caracte de estas tecnologías son distintas, por lo que es necesario determinar la característica de cada portadora para lograr un funcionamiento correcto. Para los efectos de este trabajo, en Chile hay bandas A(1850$1865 \mathrm{MHz}), \mathrm{B}(1870-1885 \mathrm{MHz}), \mathrm{C}(1895-2010 \mathrm{MHz})$, ocupados por empresas comerciales, en sistema GSM en las bandas A y C, y CDMA en la banda B. Más detalles se dan en [7].

\section{Receptor de la estación base}

Este receptor debe: recibir la señal y convertirla desde su frecuencia original a frecuencia de banda base; y captar la información de la señal recibida, hacerla llegar al PC, para que éste, mediante sus herramientas de análisis, la transforme en datos que permitan realizar la correcta configuración del sistema, de acuerdo a la tecnología de la portadora recibida. Se adoptó el esquema de recepción indicado en la Fig. 2, que consta de siete etapas de etapas funcionales:

Etapa 1. Consta de la antena receptora (1850-2010 $\mathrm{MHz}$ ) con filtros pasabandas (Chebyshev) para separar las bandas A, B y C.

Etapa 2. Es la parte que le entrega la información al PC para que este pueda configurar el sistema correcto. Consta de tres detectores de señal, que se encuentran a la salida de los filtros pasabanda. Estos detectan de qué filtro está saliendo la señal y envían esta información al PC para que él configure el resto del sistema.

Etapa 3. Consta de un conmutador inteligente que cambia de posición, de acuerdo con la orden dada por el
PC, hacia la guía de la banda que está siendo recepcionada

Etapa 4. Consta de un amplificador de bajo ruido (LNA) y un oscilador local (LO). El LO baja la frecuencia de entrada a una frecuencia intermedia (FI) más accesible al trabajo posterior. La frecuencia de oscilación se la entrega el PC, ya que depende de la banda receptora. Los valores son: $1615 \mathrm{MHz}$ para la banda A, $1635 \mathrm{MHz}$ para la banda B y de $1660 \mathrm{MHz}$ para la banda $\mathrm{C}$.

La salida de este bloque mezclador será (asumiendo que la entrada es de la forma de un seno y la oscilación del LO es de la misma característi

$$
\left.\mathrm{S}=1 / 2 \operatorname{COS}\left(\mathrm{f}-\mathrm{f}_{\mathrm{LO}}\right)-\operatorname{COS}\left(\mathrm{f}+\mathrm{f}_{\mathrm{LO}}\right)\right]
$$

donde: $\mathrm{f}$, frecuencia de entrada, y fo, frecuencia del oscilador.

El LNA (Low Noise Amplifier) amplifica las señales en un espectro de frecuencia determinado, sin aumentar la potencia de las señales de baja frecuencia, las que por lo general son de ruido blanco.

Etapa 5. Consiste en un filtro pasabajos, de frecuencia de corte de $250 \mathrm{MHz}$, que filtra las señales de más alta frecuencia, representada por $\operatorname{COS}\left(\mathrm{f}+\mathrm{f}_{\mathrm{LO}}\right)$, con lo cual la señal más alta en la salida de esta etapa tiene una frecuencia de $\left(\mathrm{f}-\mathrm{f}_{\mathrm{LO}}\right)$, cuya mayor frecuencia será de 250 MHz.

Etapa 6. En esta etapa se obtiene la portadora en banda base. Para ello se emplea un segundo oscilador local, de $205 \mathrm{MHz}$, con lo cual resultan frecuencias de $45 \mathrm{MHz}$ y $455 \mathrm{MHz}$. La señal es amplificada (en un LNA) y filtrada para obtener la portadora de banda base de $45 \mathrm{MHz}$.

Etapa 7. Consiste en una conversión análoga digital (ADC) para el procesamiento digital (DSP) y envío a la MTSO (Mobile Telephone Switching Office). La frecuencia mínima de mues treo debe ser de $90 \mathrm{MHz}$.

\section{Transmisor de la estac ión base}

El transmisor de la estación base, que se muestra en la Fig. 3, debe: recibir las señales del DSP y enviarlas al espacio a la frecuencia (RF) necesaria para que la estación móvil la pueda procesar correctamente; y convertir a forma análoga la señal de banda base digital del DSP. 


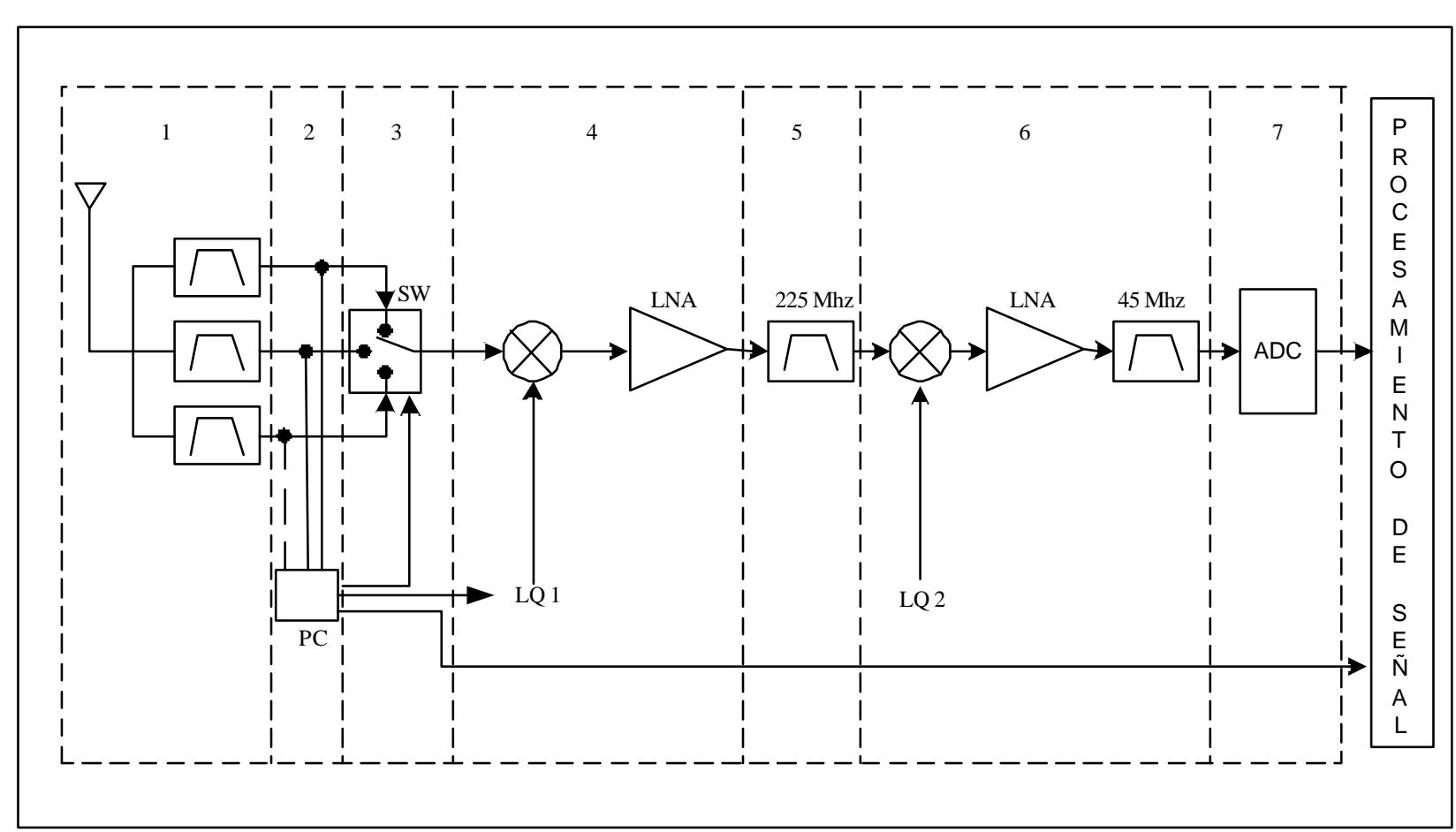

Fig. 2.- Esquema de recepción para la esta

En estos dos procesos se debe considerar que las señales a transmitir son de distinto ancho de banda y que se transmiten por distintas bandas de frecuencia: A y C, de 74 canales de $200 \mathrm{KHz}$ de ancho de banda en GSM; banda $\mathrm{B}$, de 11 portadoras ancho de banda de $1,23 \mathrm{MHz}$ cada una, en CDMA.

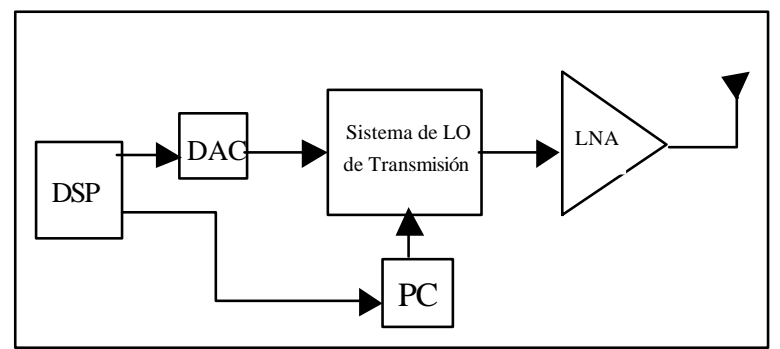

Fig. 3.- Esquema de transmisión de una estación base

La parte de transmisión de una estación consta amente de tres dementos activos: DAC (Digital Analog Conversor), LNA, y LO. Este último recibe información procedente del PC para su configuración. El conversor digital/análogo convierte a forma análoga la señal digital entregada por el DSP. Estas señales ogas serán de una frecuencia similar a la frecuencia de banda base.

\section{Sistema de LO de transmisión}

Recibe la señal de salida en banda base del DAC y sube la frecuencia de ésta, de acuerdo a la tecnología (CDMA o GSM) y ubicación de la estación móvil de destino.
Para ello, el sistema de LO consta de parámetros modificables, que son proporcionados por el PC. Este último, manejando datos que le entrega el DSP y usando las herramientas de desarrollo y análisis de datos, determina los valores y rangos a los que deben oscilar los LO.

LNA. Amplifica la potencia de la señal de salida, para que ella cumpla con los estándares correspondientes.

DSP. Es un elemento computacional que trabaja con señales. DSP es la matemática, el algoritmo, y las cnicas utilizadas para manipular estas señales después que han sido convertidas en digitales. La ventaja del DSP es su gran flexibilidad y su fácil reprogramación para el cumplimiento de otras funciones. Puede ser programado para tareas tales como diseño de filtros, multiplexado, conversores A/D y D/A, convolución, procesamiento de audio, y otros.

En este caso, el DSP cumplirá diferentes funciones, dependiendo del tipo de señal que esté recibiendo la estación base, es decir, GSM o CDMA. Entre estos están: detección de errores (código de redundancia cíclica, CRC); código de convolución y algoritmo de Viterbi; entrelazamiento; modulación y demodulación (GMSK para GSM y QPSK para CDMA); mezcla de datos, para seguridad y privacidad; subcanal de control de potencia (de salida de la estación móvil en el enlace ascendente (uplink); extensión ortogonal del canal (en que se agrega un código Walsh para individualizar cada 
canal de tráfico en el enlace descendente); separación en cuadratura (para dar una identidad a la estación base). Más detalles aparecen en [7].

GSM. En el canal de bajada la señal es enviada de la estación base hacia la estación móvil. El procesador multimedia y el procesador de ondas, toman la señal de la MTSO y le aplican un código de redundancia cíclico (CRC), un código de convolución y entrelazamiento para modular en el módem. En la Fig. 4 se presenta un esquema de este proceso. El proceso en el canal de subida es ilustrado en la Fig. 5.

CDMA. En el canal de bajada, las ráfagas de datos provenientes de la MTSO son enviadas al móvil, como se ilustra en Fig. 6. El proceso en el canal de subida es ilustrado en la Fig. 7.

\section{SIMULACIÓN}

En esta sección se presenta el esquema desarrollado, en MATLAB, para la simulación de la estación base dise aron las bibliotecas de CDMA, Comunicaciones y SIMULINK. En aquellos casos en que MATLAB no cuenta con bloques funcionales, como para la simulación de GSM, se desarrollaron programas capaces de cumplir con las funciones necesarias de modulación y demodulación, entrelazamiento, código Viterbi y convolución, y CRC. En la Fig. 8 se presenta un esquema con lo más importante de la simulación.

En las Figs. 9-12 se ilustran bloques de simulación del PC, sistema de banda base y del DSP en enlaces ascendente y descendente, respectivamente.

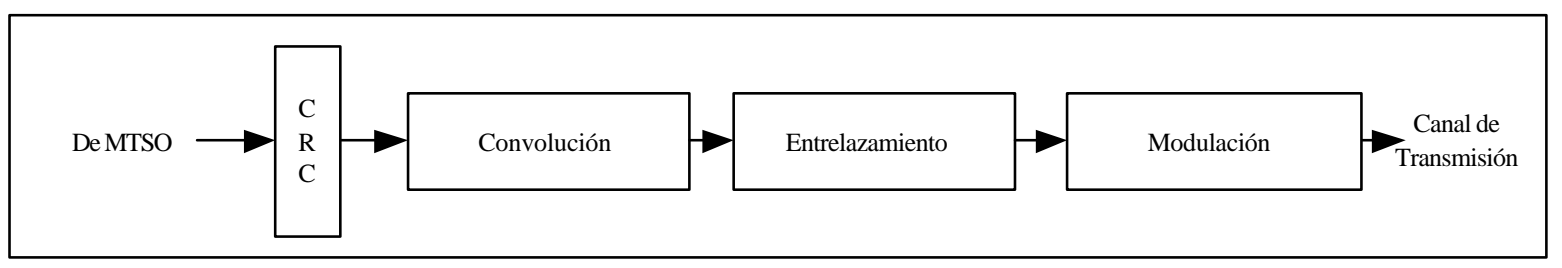

Fig. 4.- Etapas del DSP para bajada en GSM

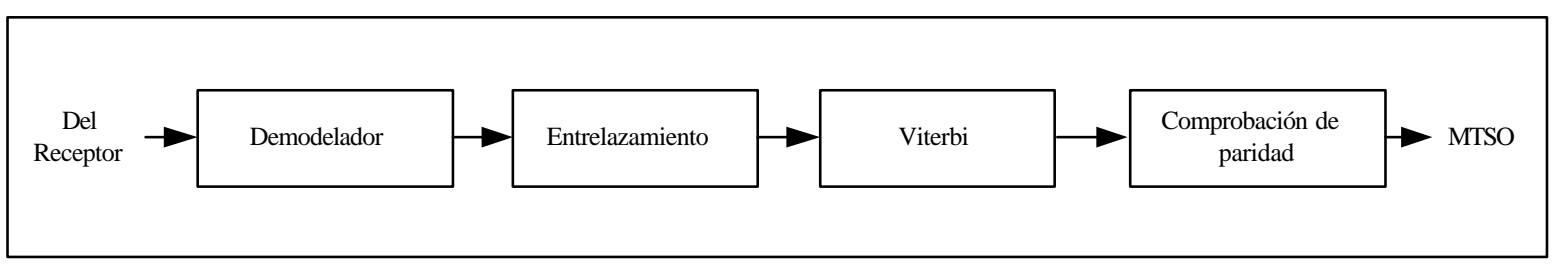

Fig. 5.- Esquema de señal GSM de subida

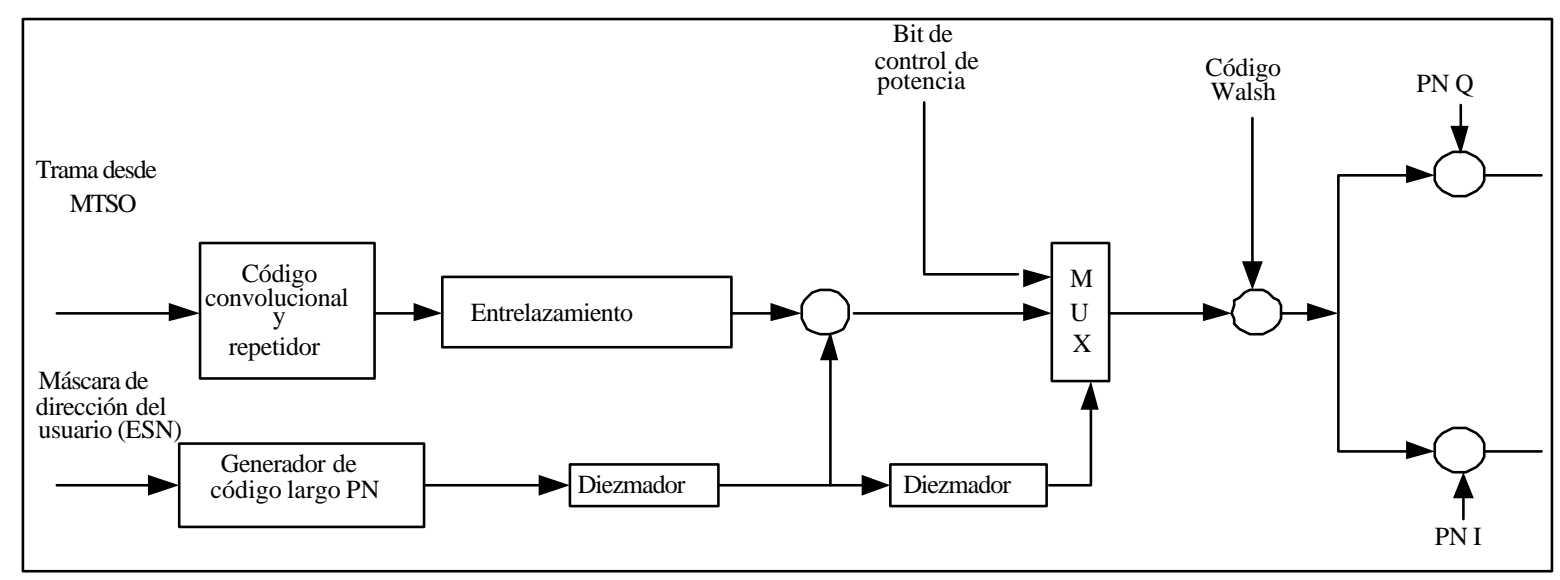

Fig. 6.- Estructura del DSP para CDMA de bajada 


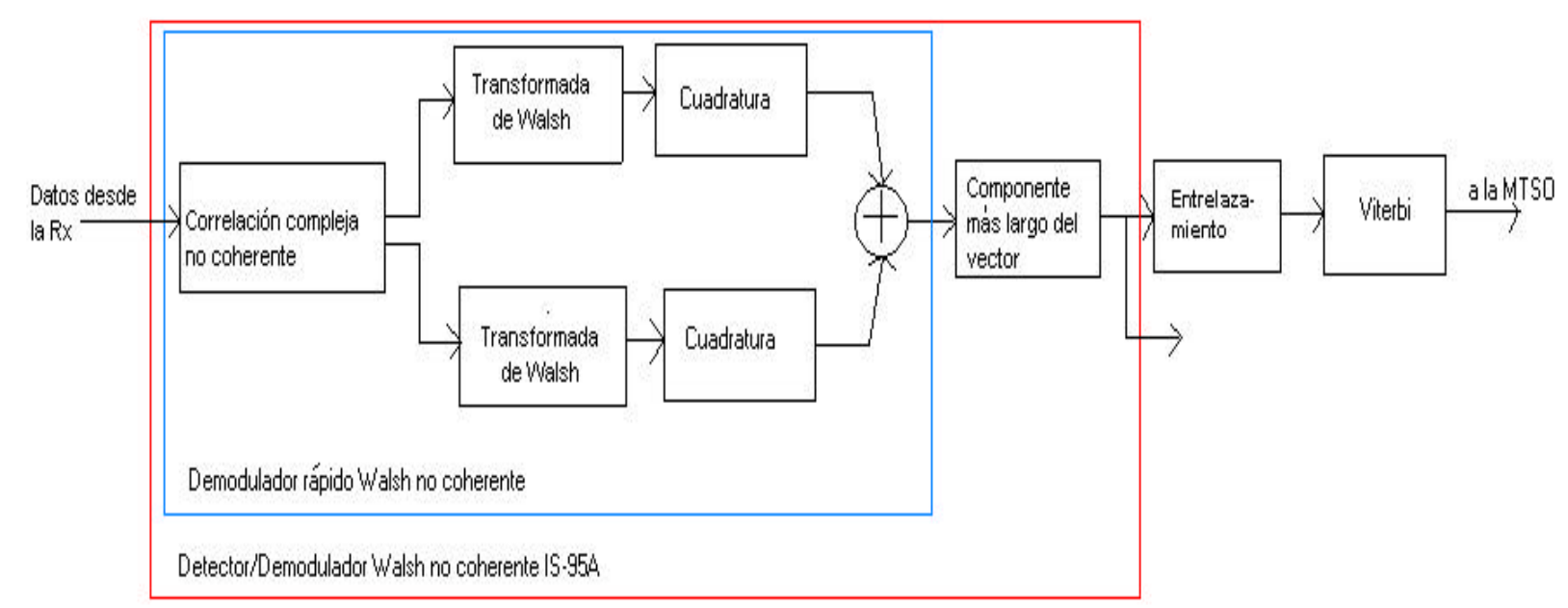

Fig. 7.- Estructura del DSP en CDMA de subida

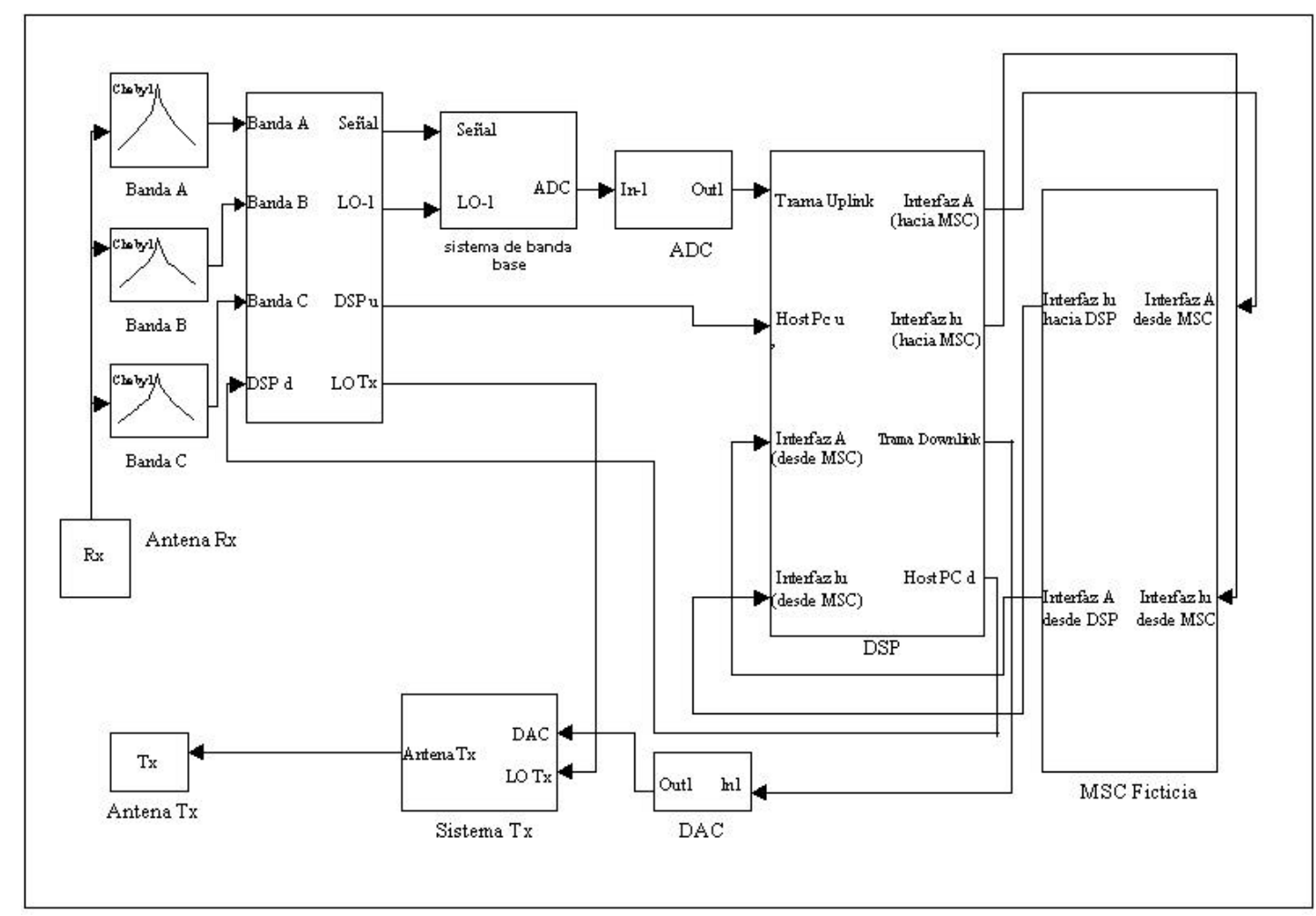

Fig. 8.- Esquema simulado de la estación base 


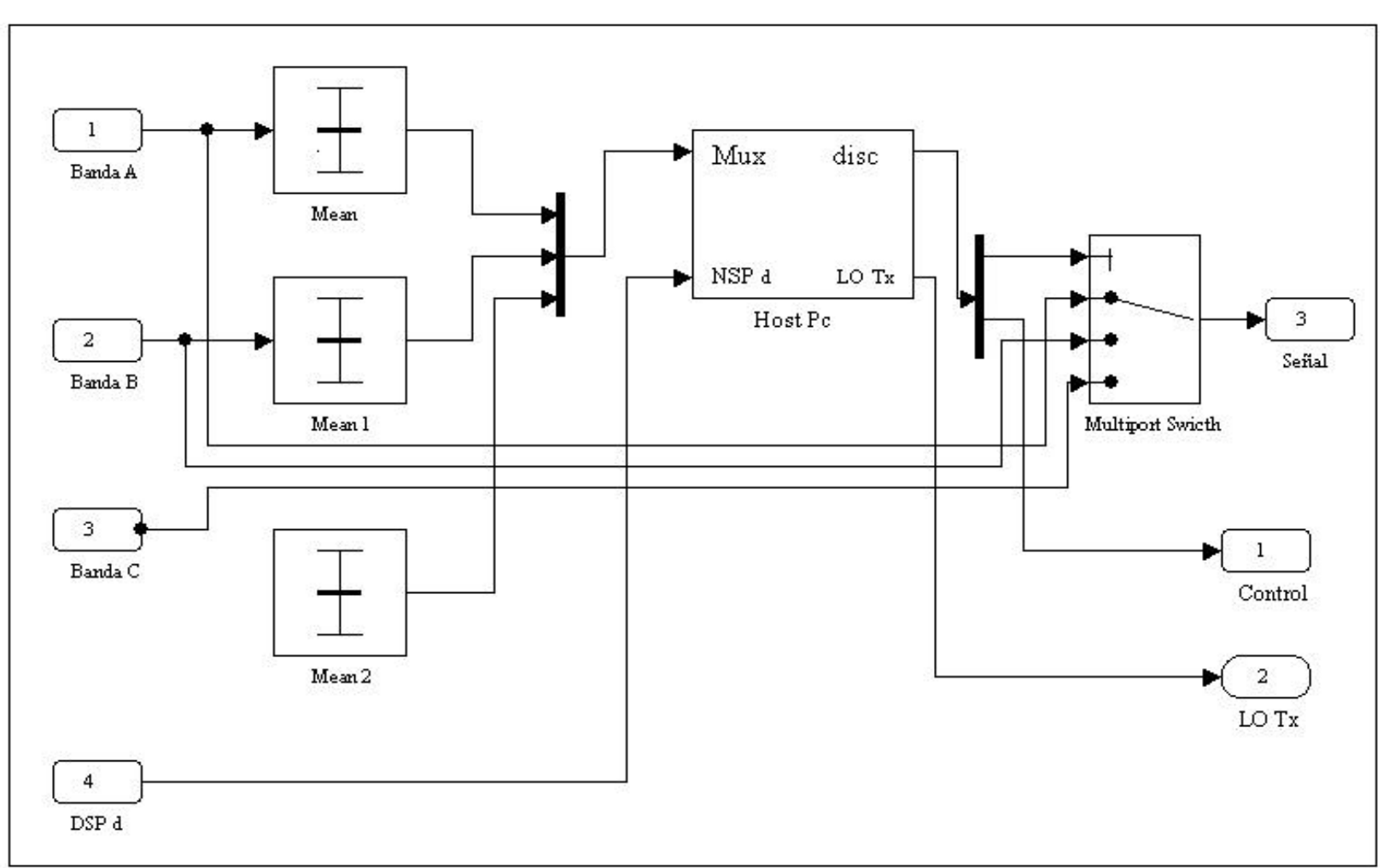

Fig. 9- Esquema interno del PC en la simulación

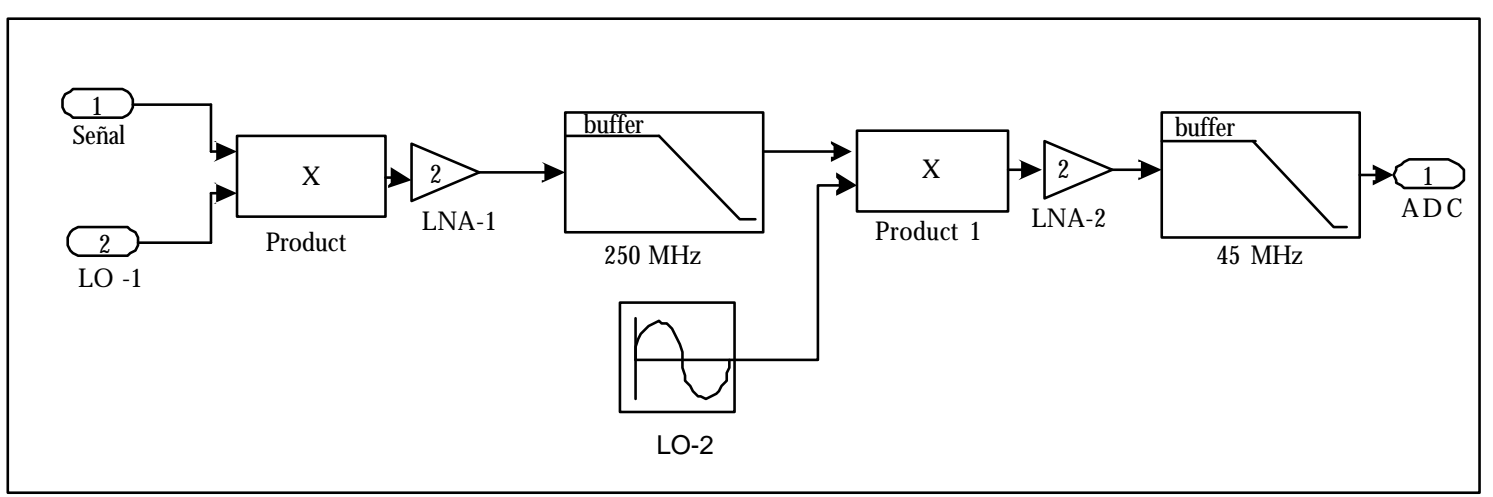

Fig. 10.- Esquema interno del sistema de banda base

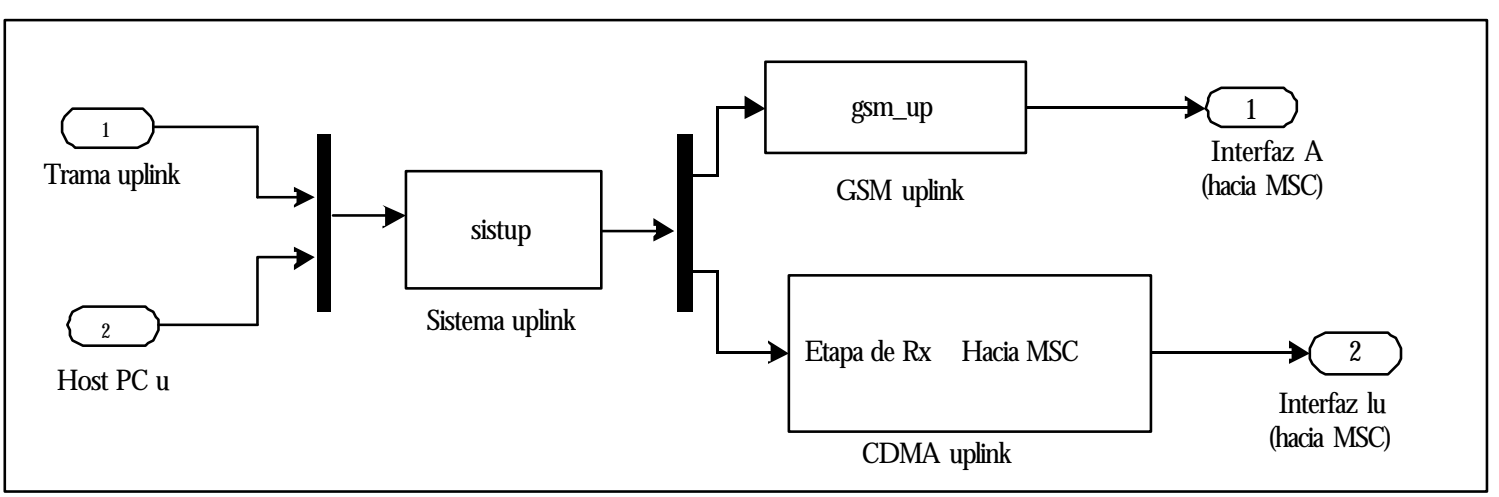

Fig. 11.- Esquema interno del DSP para subida (uplink) 


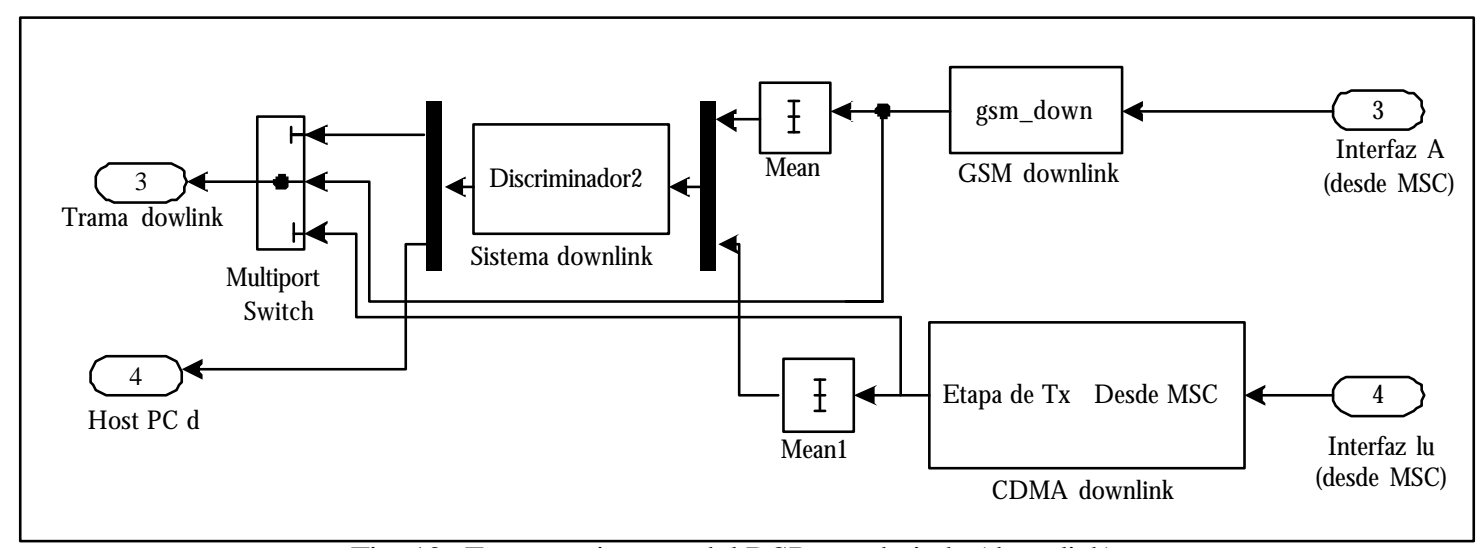

Fig. 12.- Esquema interno del DSP para bajada (downlink)

\section{CONCLUSIONES}

La tecnología de Software Radio. La simulación resultó exitosa en todos los aspectos, cambia los esquemas tradicionales de hardware y software, flexibiliza y abre nuevos niveles de servicios y economías de escala, gracias a que permite aplicaciones con múltiples bandas de accesos y variados modos de operación, reducir el hardware necesario para estas tareas y complementarlo en el uso compartido del mismo. En este trabajo se consideró el problema de uso compartido de tecnologías GSM y CDMA empleando Software Radio. También posibilita el integrar nuevos modos de accesos, agregando sólo software o un mínimo de hardware junto a líneas de programa. Esto último tiene significativa importancia en los saltos de generación de las distintas tecnologías, ya que, por lo general, el hardware es el mismo, pero agregando líneas de programa se puede llegar a realizar los servicios de la nueva generación.

En estos tipos de sistemas es vital el complementar el hardware y software, buscando y logrando de esta manera flexibilizar el uso del primero, que en general es el más costoso y difícil de adaptar. Es el caso de la etapa en que se discrimina el tipo de señal de entrada a la estación base, en la que un sencillo sistema de filtros unido a unas líneas de programa logran dicho objetivo, con lo que se logra configurar el resto de la recepción de la señal. Asimismo, en las etapas en que el sistema baja la frecuencia de entrada en recepción, o la sube en transmisión, se logra un ahorro considerable de hardware reemplazando éste por software.

En un adecuado sistema de recepción y transmisión, si se acompaña éste de un conveniente software que reconozca el tipo de entrada y cargue los programas indicados en la etapa de DSP, este puede crecer y, procesar distintas señales con sólo agregar el software pertinente. También se aprecia que en la etapa de DSP pueden coexistir diversos lenguajes de programación, y distintos sistemas operativos.

Si bien esta tecnología está acotada por la capacidad de las máquinas y la velocidad con que éstas pueden captar, capturar y procesar las distintas entradas y salidas del sistema, ello se considera una dificultad menor, ya que son precisamente estos elementos y tecnologías bs que se están desarrollando con mayor velocidad.

\section{REFERENCIAS}

[1] T. Rappaport; Wireless Communications, Upper Saddle River: Prentice Hall, 1996.

[2] R. Kurupillai, M. Dontanusetti, and F. Cosentino; "Wireles PCS", New York: McGraw-Hill, 1997.

[3] J. Mitola; "The Software Radio Architecture", IEEE Communications Magazine, pp. 26-38, May 1995.

[4] S. P. Reichart et al.; "The Software Radio Development System", IEEE Personal Communications, pp. 20-24, August 1999.

[5] K. C. Zangi., and D. Koilpillai, "Software Radio Issues in Cellular Base Stations", IEEE Journal on Selected Areas in Communications, Vol. 1, N ${ }^{\circ} .4$, pp. 561-573, April 1999.

[6] A. Pérez-Neira et al.; "Smart Antennas in Software Radio Base Station", IEEE Communications Magazine, pp. 166-173, Feb. 2001.

[7] J. G. Busqués V. y S. H. E. Reyes; "Diseño y simulación de una estación base GSM/CDMA utilizando Software Radio", Memoria de Título, U.T.F.S.M, Valparaíso, Chile, 2002. 